\title{
Energy Absorption Enhancement by Unit Cell Angle Grading for Sandwich Panels with Auxetic Core
}

\author{
OANA ALEXANDRA MOCIAN, DAN MIHAI CONSTANTINESCU* ${ }^{*}$, FLORIN BACIU, \\ ANDREI INDRES
}

University Politehnica of Bucharest, Department of Strength of Materials, 313 Splaiul Independenței, 060042, Bucharest, Romania

\begin{abstract}
Architectured structures, particularly auxetic materials, have demonstrated encouraging applications in energy absorption as they facilitate the customization of their structural response. Specific geometries of unit cells can thus be tailored for particular needs due to recent progress in additive manufacturing techniques. This paper experimentally studies how the grading of the cell unit angle of an auxetic core in a sandwich panel affects its energy absorbing capability and structural response. 3D printed sandwich panels with uniform and graded auxetic cellular core were tested under quasistatic compression. The results show that sandwich panels with graded core exhibit much better energy absorption capabilities with higher plateau stress and densification strain. This indicates that, by appropriately controlling its geometry, auxetic structures can show further potential as core in sandwich panels for energy absorption applications.
\end{abstract}

Keywords: lightweight, sandwich panel, auxetic cellular structure, graded unit cell, 3D printing

\section{Introduction}

Recently, many industries have been widely using cellular materials and structures for their favorable mechanical properties and increased energy absorption performances in addition to their light weight [14]. Even though conventional cellular materials like foams exhibit excellent energy dissipation properties, low manufacturing costs and low densities, their energy absorption performances cannot be further enhanced, as their manufacturing techniques usually result in random unit cells and irregular topology. To improve energy absorption performances and other mechanical properties, new geometries of periodic cellular structures were designed [5-8]. The complexity of these novel structures increased over the years, presenting a critical challenge of fabrication that traditional manufacturing techniques are no longer suited for. Having the ability to produce intricate cellular structures in an economical and controllable way, additive manufacturing (AM) or three-dimensional printing (3DP) allows the fabrication of features that were previously unreachable. Thus, the cellular structure can achieve particular mechanical properties by controlling the architecture of the repeating unit cell $[9,10]$. Moreover, some AM technologies allow the fabrication of an integral structure with differential mechanical properties [11,12].

The design of cellular structures is a unit cell-based design arranged in two- or three-dimensional arrays. Among the different types of cellular structures, auxetic structures, are some of the most promising ones in terms of specific mechanical properties. They are typically known for their negative Poisson's ratio, meaning that, likewise conventional materials, they expand/contract laterally under uniaxial traction/compression. This behavior leads to improved shear stiffness [13,14], increased indentation resistance [15-16], remarkable fracture toughness [17] and high energy absorption capabilities $[18,19]$. Among all these, their light weight and energy absorption properties make these structures particularly attractive and useful in some modern applications. They can be successfully used as cores in sandwich panels for ballistic protection in body and vehicle armor applications [20]. Also, in sports, auxetic structures can be used as flexible pads to reduce impact severity for different body parts (e.g., head, elbow, leg) [21].

\footnotetext{
*email: dan.constantinescu@upb.ro
} 
The use of auxetic cellular structures for energy absorption applications has received much attention not only in industry, but also in research where an important number of valuable works has been published recently. A lot of effort has been put to analyze the static and dynamic energy absorption capabilities of auxetic structures, through both experimental and numerical methods. For example, Tan et al. [22] parametrically studied the deformation modes and energy absorption capabilities of re-entrant hierarchical hexagonal honeycombs and demonstrated that both their proposed solutions exhibit an improved crushing performance. To further improve the energy absorption performance in impact, Wang et al. [23] designed and studied a novel auxetic structure named re-entrant star-shaped honeycomb (RSH) and showed, through both experimental and numerical simulation, that RSH shows excellent impact resistance, compared with the classical re-entrant honeycomb $(\mathrm{RH})$ with same cell wall thickness. Using the finite element method, Qi et al. [24] examined the performance of a new protective system using auxetic honeycomb-cored sandwich panels for mitigation of shock loads from detonations of high explosives. They found that the proposed auxetic panels increased the energy absorption capacity of the monolithic steel plate by a factor of 2.5 changing its deformation pattern.

Most of the cellular structures studied to date have homogenous materials and uniform cells. To further improve the energy absorption capacity of these type of structures, the concept of graded structures came into attention for many researchers, as it has been indicated that in this manner the deformation mechanisms can be controlled and thus, by adopting a proper gradient strategy, a better energy absorption performance could be achieved. For example, Li et. al. [25], showed that, under high crushing velocity, piecewise linear graded honeycombs have better energy absorption capacity but low impact resisting behavior than the uniform honeycombs. Ajdari et al. [26] investigated by using detailed finite element models the in-plane dynamic crushing of two-dimensional honeycombs with both regular and irregular cell structure. By applying a gradual change of the cell wall thickness, a density gradient in the direction of crushing was introduced which proved to enhance the energy absorption of honeycombs at early stage of crushing.

In this study, we propose a unit cell angle grading for the auxetic core of a sandwich panel as to enhance its energy absorption capacity while keeping the stiffness and strength between acceptable limits. We used 3D printing to manufacture sandwich panels with auxetic core of acceptable quality. The resulting sandwich panels were subjected to uniaxial compression. Based on the experimental results, the mechanical behavior and energy absorption efficiency of the proposed graded core sandwich panel and the conventional uniform core sandwich panels were compared and discussed.

\section{Materials and methods}

\subsection{Experimental methodology}

\subsubsection{Geometry and fabrication}

The auxetic cores were generated using the unit cell shown in Figure 1.

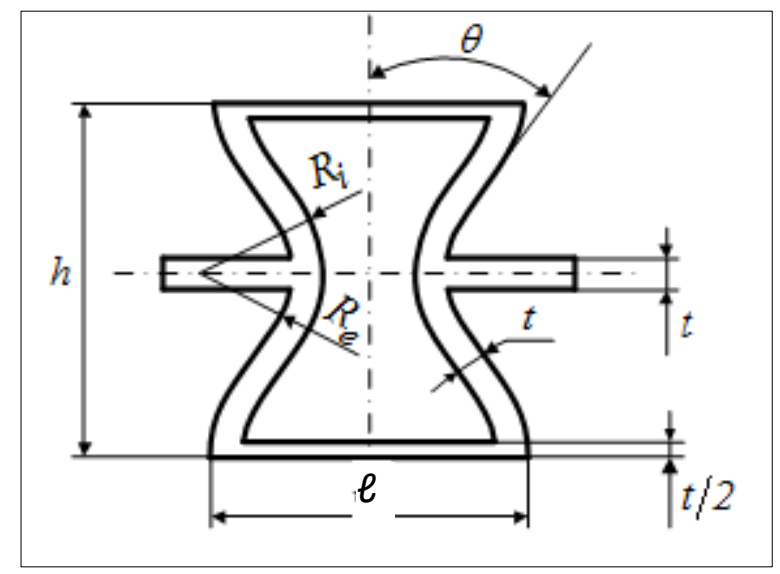

Figure 1. Unit cell geometry of auxetic core 
The geometry of the unit cell is defined by the following parameters: the internal cell angle $\theta$, the cell wall thickness $t$, the height of the unit cell $h$, the length of the unit cell $\ell$, the internal cell radius $R_{i}$ and the external cell radius $R_{e}$. The grading effect on the auxetic core was obtained by varying the internal cell angle $\theta$ along the height of the core. Three types of unit cells were thus obtained, as presented in Table 1.

Table 1. Geometrical parameters of unit cells used in the graded auxetic core

\begin{tabular}{|c|c|c|c|c|c|c|}
\hline \multirow{2}{*}{ Unit cell type } & \multicolumn{7}{|c|}{ Parameter } \\
\cline { 2 - 7 } & $\theta[\mathrm{deg}]$ & $t[\mathrm{~mm}]$ & $h[\mathrm{~mm}]$ & $R_{i}[\mathrm{~mm}]$ & $R_{e}[\mathrm{~mm}]$ & $\ell[\mathrm{mm}]$ \\
\hline UCT 1 & 5 & 5 & 6 & 2 & 1.5 & 8.596 \\
\hline UCT 2 & 20 & 5 & 6 & 4 & 3.5 & 9.425 \\
\hline UCT 3 & 35 & 5 & 6 & 6 & 5.5 & 10.434 \\
\hline
\end{tabular}

Based on these types of unit cells three types of sandwich panels were fabricated (Figure 2). Two of them have a uniform auxetic core with unit cell type 1 and 3 and one has a graded core that has all three types of unit cells. All of them have the same dimensions $40 \times 40 \times 40 \mathrm{~mm}$. The facesheets of the sandwich panels have $2 \mathrm{~mm}$ thickness, while the core has $36 \mathrm{~mm}$ thickness. All cores, uniform or graded, have six cells, in both horizontal and vertical direction.

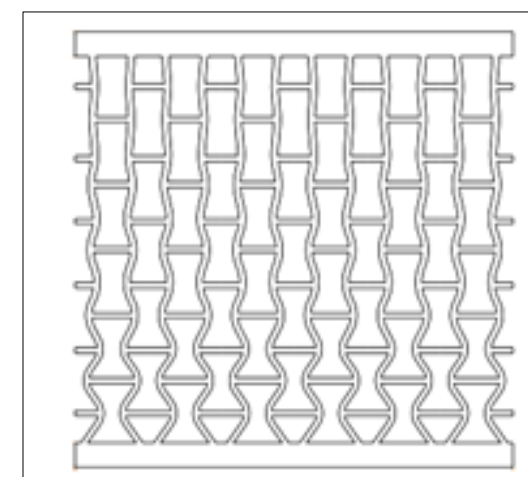

a)

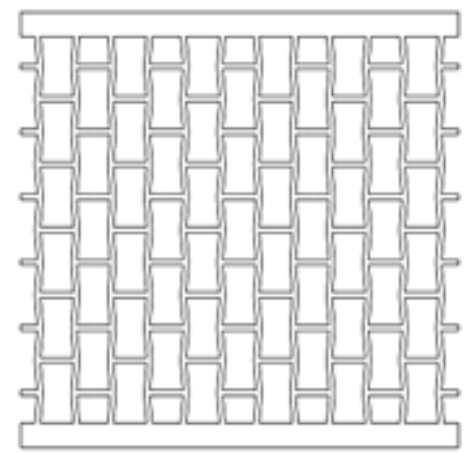

b)

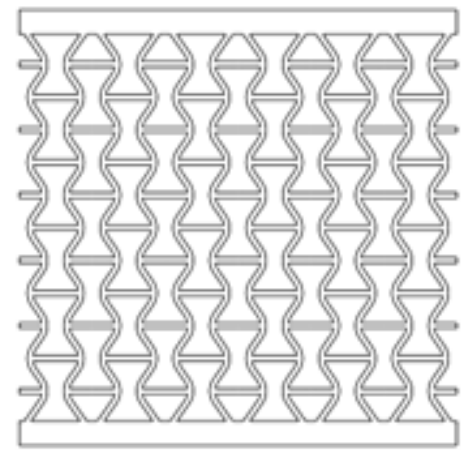

c)

Figure 2. Sandwich panels configuration: a) graded auxetic core (graded core in the legends of the figures to follow); b) uniform auxetic core - unit cell type 1 (UCT 1);

c) uniform auxetic core - unit cell type 3 (UCT 3)

The configuration of the sandwich panels was first created using CATIA V5 software, then the stereolithography file (.stl) was outputted into a Fused Deposition Modeling (FDM) 3D printer (Ultimaker 3 Extended) to realize the auxetic core structure and the facesheets of the sandwich panels. FDM is one of the most used and affordable 3D printing technology where melted filaments of thermal plastics are selectively deposited in a predetermined path, layer-by-layer. A polylactic acid (PLA) material filament with a density of $1.24 \mathrm{~g} / \mathrm{cm}^{3}$ together with a $0.25 \mathrm{~mm}$ extruder were used to build, with an infill topology of $100 \%$, both the core and the facesheets of the sandwich panels. Melted PLA layers of $0.1 \mathrm{~mm}$ height were deposited on the printing plate at a printing temperature of $190^{\circ} \mathrm{C}$ and a printing speed of $30 \mathrm{~mm} / \mathrm{s}$. The printing building direction was parallel to the height of the specimen placed on the printing bed. The saturation of the curing process for all printed sandwich panels was attained by keeping them at room temperature for at least one week after the fabrication process.

\subsubsection{Testing method}

Uniaxial compression of the printed sandwich panels was undertaken using an Instron 8872 universal testing machine. The $25 \mathrm{kN}$ load cell of the system allowed to record data with a good accuracy. The sandwich panels were placed on a fixed metal plate, and they were compressed up to densification, 
normal to the fabrication direction, by a moving metal plate at a constant speed of $2 \mathrm{~mm} / \mathrm{min}$. The compressive force and displacement of the moving platen were recorded. Because of the long time needed to print one sandwich panel (almost $25 \mathrm{~h}$ ) only two samples of each design were tested.

\section{Results and discussions}

The mechanical properties and energy absorption capabilities of the uniform and graded auxetic core sandwich panels were analyzed based on the uniaxial compression tests.

\subsection{Mechanical behavior}

The mechanical behavior is analyzed using the stress-strain curves. This are calculated based on the force-displacement recorded data and considering the initial cross-sectional area and initial height of the specimens. The resulting engineering stress-strain curves are presented in Figure 3.

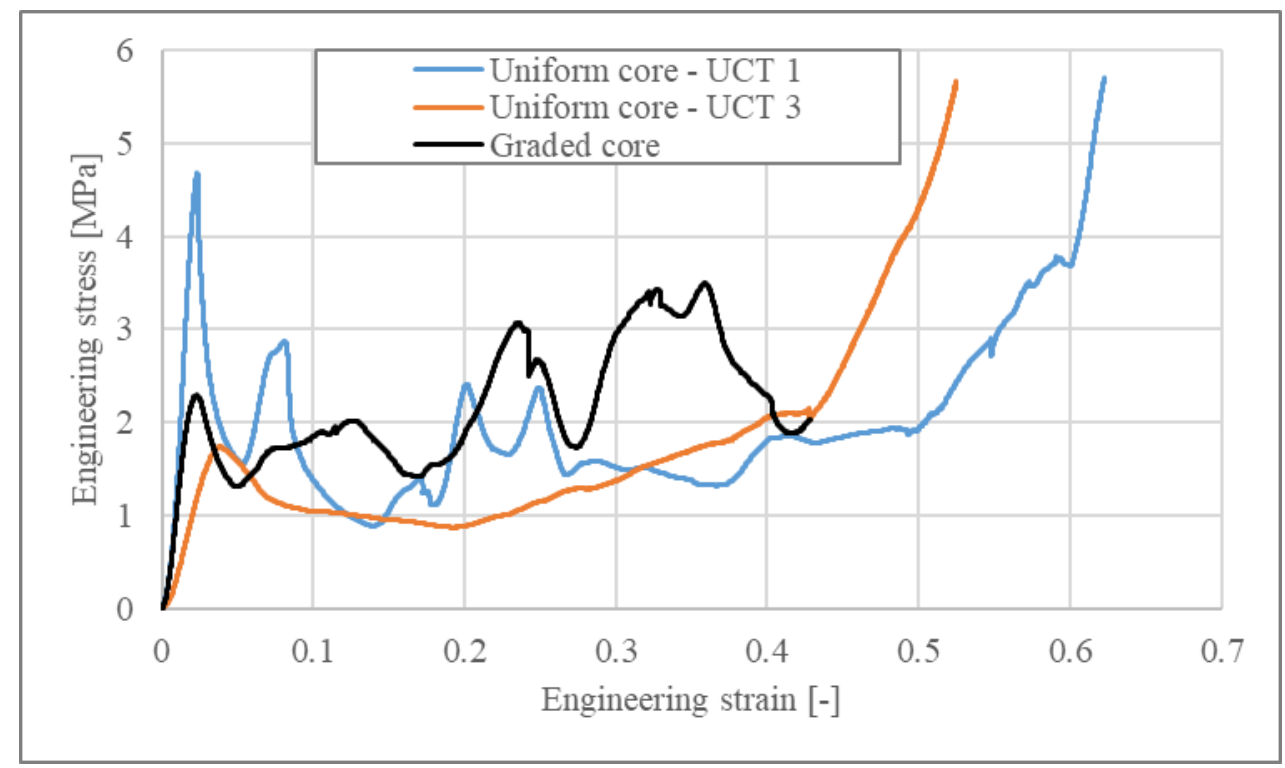

Figure 3. Experimental quasi-static compressive stress-strain curves for sandwich panels with uniform and graded auxetic core

Similar to conventional cellular materials, the stress-strain curves of all three sandwich panels can be roughly divided into three distinct zones: pre-collapse, post-yield plateau and densification [27]. The first zone corresponds to the initial portion of the stress-strain curve before the first peak stress and ends when yielding becomes widespread in many cells. The initial peak stress is very important especially if the sandwich panels are used in body or vehicle armor applications where they should absorb most of the impact energy and transmit the least amount of stress possible. Immediately after reaching the initial peak a drop in stress occurs and the plateau regime starts. This is the part of the stress-strain curve where most of the impact energy is absorbed and consequently is the most significant in establishing the energy absorption performances of a structure. Thus, a protracted, high, and stable stress response before final densification (steep rise in stress) is desirable [27].

As illustrated in Figure 3, the sandwich panel with uniform auxetic core UCT1 has the highest peak stress, followed by the most severe drop of stress and a plateau with a sequence of undesired fluctuation that tend to reduce its energy absorption capacity significantly. The severe drop of stress is caused by a severe buckling of the cells' walls on a direction that goes from the lowest left cell up almost to the highest right cell of the auxetic core. In comparison, the sandwich panel with uniform auxetic core UCT3 has an initial peak stress almost three time smaller and exhibits stable post-yield response. Though, the stress values in the plateau zone are significantly lower and thus the total absorbed energy is smaller. For the sandwich panel with graded core the initial peak stress is a little higher than in the case of the 
sandwich panel with uniform core UCT3, enough to transmit less stress further but to give at the same time sufficient rigidity to the sandwich structure. The plateau zone has also important fluctuations, but unlike the sandwich panel with uniform core UCT1, the subsequent stress peaks have a bigger value than the initial one. This happens because, due to different cell topology, the core fails layer by layer and pre stages of densification occur. The first cells to fail are the ones with the biggest value of the internal cell angle, namely UCT3. This pre stages of densification tend to increase the energy absorption capacity of the sandwich panel with graded core. The last part of the stress-strain curves indicates that sandwich panels with unit cells that have the highest internal cell angle have smaller densification strains.

\subsection{Energy absorption characteristics}

Energy absorption is one of the most important assessments of performance for sandwich panels with cellular cores. Based on the experimental results, the energy absorption (E) is calculated as the area under the stress-strain curve from initial loading up until densification strain $\left(\varepsilon_{\mathrm{d}}\right)$ :

$$
E=\int_{0}^{\varepsilon_{d}} \sigma(\varepsilon) d \varepsilon
$$

There are many different methods available to determine the densification strain. One of the most common used, calculates the densification strain based on the energy absorption efficiency. According to this, the densification strain is the point where the last local maximum energy absorption efficiency occurs [28]. The energy efficiency $(\eta)$ is described as the ratio between the absorbed energy up to a given strain and the corresponding nominal stress [28]:

$$
\eta=\frac{\int_{0}^{\varepsilon} \sigma(\varepsilon) d \varepsilon}{\sigma(\varepsilon)} .
$$

The calculated energy efficiency-strain curves for the examined sandwich panels are presented in Figure 4.

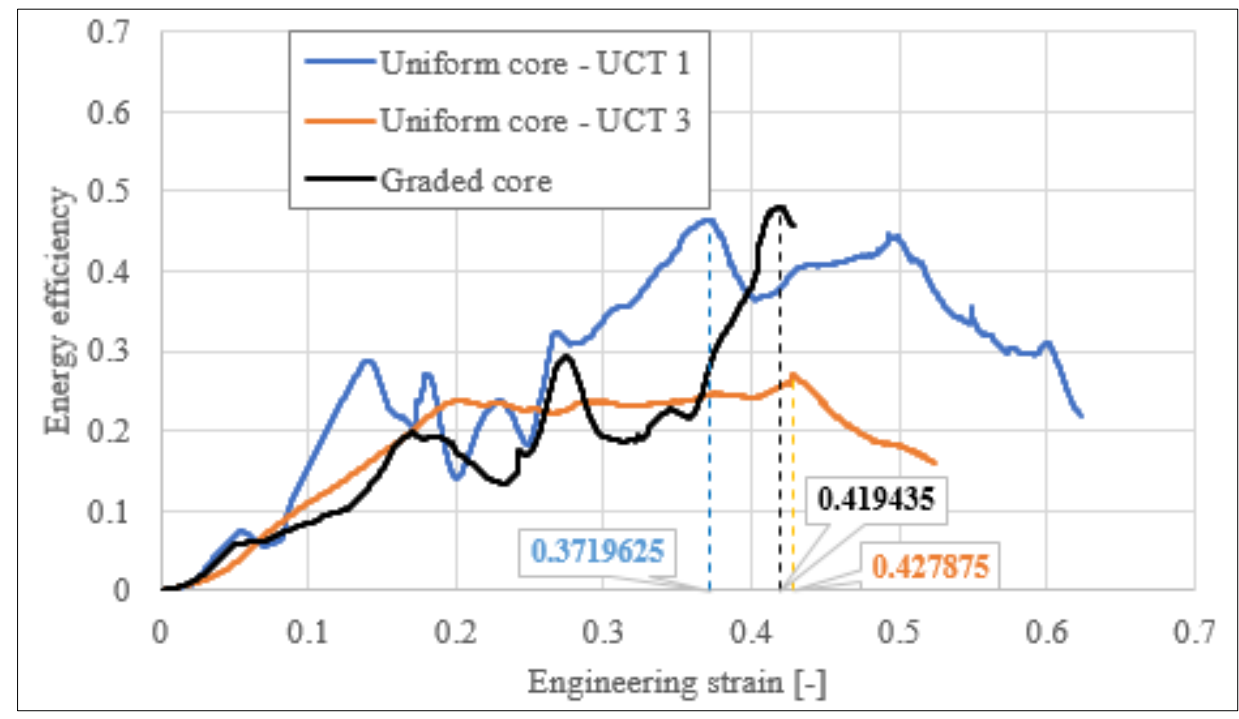

Figure 4. Energy efficiency-strain curves for sandwich panels with uniform and graded auxetic core

Using equation (1) and the densification strains determined above, the corresponding absorbed energy was calculated. The energy absorption-strain curves are presented in Figure 5. 


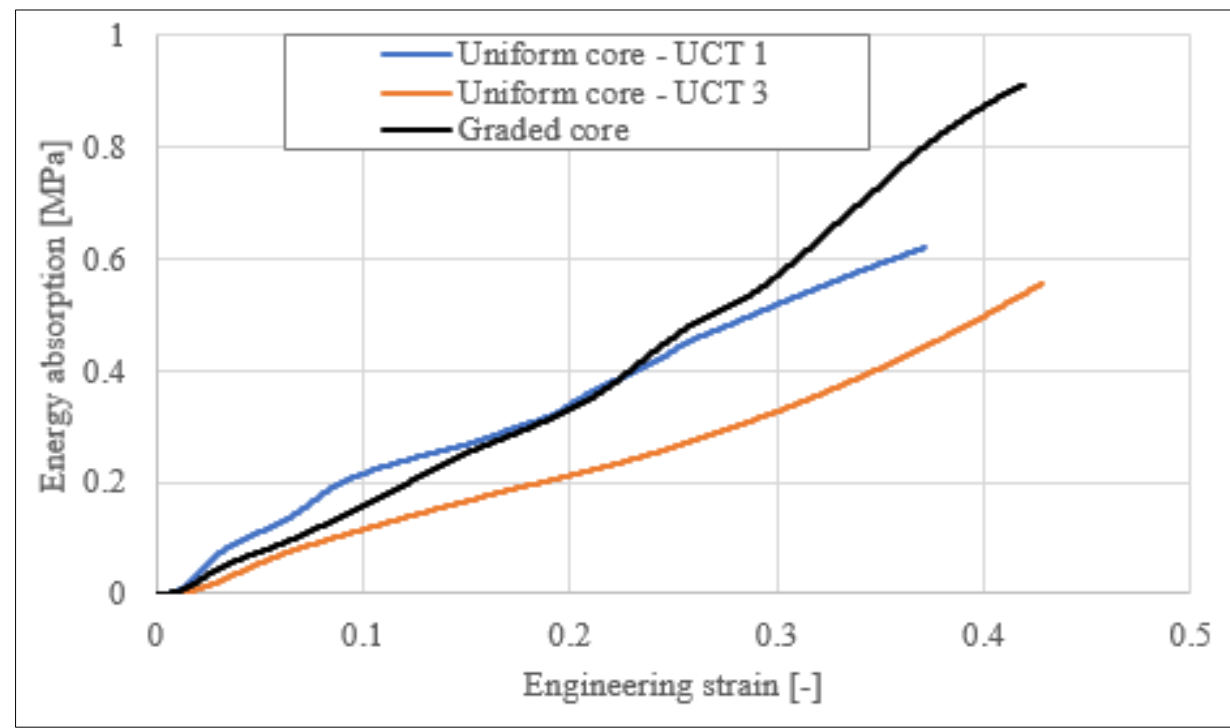

Figure 5. Energy absorption-strain curves for sandwich panels with uniform and graded auxetic core

As it can be seen from Figure 5, the sandwich panel with graded auxetic core absorbs the highest amount of energy compared to the sandwich panels with uniform auxetic core. For small strains the absorbed energy of the graded sandwich panel is slightly smaller than the uniform sandwich panel UCT1. The efficiency of the graded structure in absorbing energy becomes obviously after strains larger than 0.25 . The absorbed energy at densification strain is $0.62 \mathrm{MPa}$ for the uniform structure UCT1, $0.55 \mathrm{MPa}$ for the uniform structure UCT3 and $0.91 \mathrm{MPa}$ for the graded structure, which indicates that the graded sandwich panel has a greater energy absorption capacity.

\section{Conclusions}

Starting from the design of two sandwich panels with uniform auxetic core, the concept of a sandwich panel with graded auxetic core was developed. The objective was to enhance the energy absorption performance of these types of structures. The proposed graded sandwich structure was designed using three different cell topologies, including the ones used in the uniform core structures. The complex auxetic core structures were fabricated using 3D printing. The response of the sandwich panels to quasistatic compression was investigated through experimental means and compared in terms of mechanical behavior and energy absorption capabilities.

The results demonstrate that the sandwich panel with graded auxetic core shows enhanced energy absorption performances compared to sandwich panels with uniform auxetic core. This is mainly attributed to the fact that the graded structure has a layer-by-layer deformation mode that follows the weak to strong direction generating multiple pre stages of densification that tend to enhance its energy absorption capability. Also, the initial maximum stress value can be consistently reduced by adopting a graded structure. The graded design is easy to be manufactured using the 3D printing technology and thus promising energy absorption application potentials could be expected.

Acknowledgments: The work of Ms. Oana Alexandra Mocian was supported by the project ANTREPRENORDOC, in the framework of Human Resources Development Operational Program 2014-2020, financed from the European Social Fund under the contract number 36355/23.05.2019 HRD OP /380/6/13 - SMIS Code: 123847.

\section{References}

1. LIU, W., LI, H., YANG, Z., ZHANG, J., XIONG, C., Mechanics of a novel cellular structure for morphing applications, Aerosp. Sci. Technol., 95, 2019, 105479. 
2. VILARDELl, A.M., TAKEZAWA, A., DU PLESSIS, A., TAKATA, N., KRAKHMALEV, P., KOBASHI, M., YADROITSAVA, I., YADROITSEV, I., Topology optimization and characterization of Ti6Al4v ELI cellular lattice structures by laser powder bed infusion for biomedical applications, Mat. Sci. Eng. A. - Struct., 766, 2019, 138330.

3. VOILlAT, R., GALLIEN, F., MORTENSEN, A., GASS, V., Hypervelocity impact testing on stochastic and structured open porosity cast Al-Si cellular structures for space applications, Int. J. Impact Eng., 120, 2018, 126-137.

4. BORSELLIONO, C., DI BELLA, G., Paper-reinforced biomimetic cellular structures for automotive applications, Mater. Design, 30(10), 2009, 4054-4059.

5. ULAH, I., BRANDT, M., FEIH, S., Failure and energy absorption characteristics of advanced 3D truss core structures, Mater. Design, 92, 2016, 937-948.

6. LIU, X., ALIZADEH, V., HANSEN, C.J., The compressive response of octet lattice structures with carbon fiber composite hollow struts, Compos. Struct., 239, 2020, 111999.

7. NGUYEN, B.D., CHO, J.S., KANG, K., Optimal design of "Shellular", a micro-architectured material with ultralow density, Mater. Design, 95, 2016, 490-500.

8. WANG, Z., LI, Z., SHI, C., ZHOU, W., Mechanical performance of vertex-based hierarchical vs square thin-walled multi-cell structure, Thin Wall. Struct., 134, 2019, 102-110.

9. TOBIAS, A. CARTER, S. and. W.B., Architected cellular materials, Annu. Rev. Mater. Res., 46(1), 2016, 187-210.

10. XU, J., GAO, L., XIAO, M., GAO, J., LI, H., Isogeometric topology optimization for rational design of ultra-lightweight architected materials, Int. J. Mech. Sci., 166, 2020, 105103.

11. KOKKINIS, D., BOUVILLE, F., STUDART, A.R., 3D printing of materials with tunable failure via bioinspired mechanical gradients, Adv. Mater., 30(19), 2019, 1705808.

12. ROACH, D.J., HAMEL, C.M., DUNN, C.K., JOHNSON, M.V., KUANG, X., QI, H.J., The m4 3D printer: A multi-material multi-method additive manufacturing platform for future 3D printed structures, Addit. Manuf., 29, 2019, 100819.

13. NOVAK, N., KRSTUlOVIC-OPARA, L., REN, Z., VESENJAK, M., Compression and shear behavior of graded chiral auxetic structures, Mech. Mater., 148, 2020, 103524.

14. LIRA, C. INNOCENTI, P., SCARPA, F., Transverse elastic shear of auxetic multi reentrant honeycombs, Compos. Struct., 90(3), 2009, 314-322.

15. USTA, F., TURKMEN, H.S., SCARPA, F., Low-velocity impact resistance of composite sandwich panels with various types of auxetic and non-auxetic core structures, Thin Wall Struct., 163, 2021, 107738.

16. LI, Z., WANG, K.F., WANG, B.L., Indentation resistance of brittle auxetic structures: Combining discrete representation and continuum model, Eng. Fract. Mech., 252, 2021, 107824.

17. DONOGHUE, J., ALDERSON, K., EVANS, K., The fracture toughness of composite laminates with a negative Poisson's ratio, Phys. Status Solidi. B, 246(9), 2009, 2011-2017.

18. LINFORTH, S., NGO, T., TRAN, P., RUAN, D., ODISH, R., Investigation of the auxetic oval structure for energy absorption through quasi-static and dynamic experiments, Int. J. Impact Eng., 147, 2021, 103741.

19. ZHANG, J., LU, G., YOU, Z., Large deformation and energy absorption of additively manufactured auxetic materials and structures: A review, Compos. Part B-Eng., 201, 2020, 108340.

20. IMBALZANO, G., TRAN, P., NGO, T.D., LEE P.V.S., A numerical study of auxetic composite panels under blast loadings, Compos. Struct., 135, 2015, 339-352.

21. SANAMI, M., RAVIRALA, N., ALDERSON, K., ALDERSON, A., Auxetic materials for sports applications, Procedia Eng., 72, 2014, 453-458.

22. TAN, H.L., HE, Z.C., LI, K.X., LI, E., CHENG, A.G., XU, B., In-plane crashworthiness of re-entrant hierarchical honeycombs with negative Poisson's ratio, Compos. Struct., 229, 2019, 111415.

23. WANG, H., LU, Z., YANG, Z., LI, X., A novel re-entrant auxetic honeycomb with enhanced inplane impact resistance, Compos. Struct., 208, 2019, 758-770. 
24. QI, C., REMENNIKOV, A., PEI, L.Z., YANG, S., YU, Z.H., NGO, T.D., Impact and close-in blast response of auxetic honeycomb-cored sandwich panels: experimental tests and numerical simulations, Compos. Struct., 180, 2017, 161-178.

25. LI, Z., JIANG, Y., WANG, T., WANG, L., ZHUANG, W., LIU, D., In-plane crushing behavior of piecewise linear graded honeycombs, Compos. Struct., 207, 2019, 425-437.

26. AJDARI, A., NAYEB-HASHEMI, H., VAZIRI, A., Dynamic crushing and energy absorption of regular, irregular and functionally graded cellular structures, Int. J. Solids Struct., 48(3-4), 2011, 506516.

27. SUN, Z.P., GUO, Y.B., SHIM, V.P.W., Characterization and modelling of additively manufactured polymeric hybrid lattice structures for energy absorption, Int. J. Mech. Sci., 191, 2021, 106101.

28. LI, Q.M., MAGKIRIADIS, I., HARRIGAN, J.J., Compressive strain at the onset of densification of cellular solids, J. Cell. Plast., 42(5), 2006, 371-392

Manuscript received: 26.08 .2021 